\title{
Chromosome research-cytogenetics, genomics, chromatin, and the nucleus
}

The editors and publisher of Chromosome Research wish to thank the following persons who, in addition to members of the Editorial Advisory Board, have provided us with critical reviews of manuscripts submitted to the journal over the past year. We are especially grateful for their commitment to the success of Chromosome Research and the growth of its reputation as a leading journal in the field.

S. Abe, Sapporo, Japan

R. Adam, Tucson, AZ, USA

S. Armstrong, Birmingham, UK

P. Barker, Gaithersburg, MD, USA

H. Bass, Tallahassee, FL, USA

M. Biggiogera, Pavia, Italy

J. Birchler, Columbia, MO, USA

R. Blackman, London, UK

B. Bunnell, Tulane, LA, USA

J-P. Camacho, Granada, Spain

E. Casacuberta, Cambridge, MA, USA

A. Cavallini, Pisa, Italy

P. Chakrabarty, Baton Rouge, LA, USA

C. Chien Jan, Fargo, ND, USA

J. Dohrn, Edinburgh, UK

T. Eikbush, New York, NY, USA

C. Escude, Paris, France

T. Ezaz, Canberra, Australia

J. Fajkus, Brno, Czech Republic

M. Farman, Lexington, KY, USA

D. Fitzpatrick, Edinburgh, UK

E. Gazave, Barcelona, Spain

S. Garagna, Pavia, Italy

D. Griffin, Canterbury, UK

S. Grigoryev, Hershey, PA, USA

N. Hartmann, Liebniz, Germany

P. Hozak, Prague, Czech Republic
C. Jones, London, UK

F. Jonsson, Witten, Germany

K. Kempsell, Salisbury, UK

G. Kuhn, Bosnia, Herzegovina

R. Knast, London, UK

H. Kurahashi, Toyoake, Japan

A. Kuroiwa, Saporro, Japan

M. Labrador, Knoxville, TN, USA

J. Lee, Saskatoon, Canada

J. Loidl, Vienna, Austria

M. Lysak, Brno, Czech Republic

F. Marec, Ceske Budejovic,

Czech Republic

K. Matsubara, Nagoya, Japan

A. Maya-Mendoza, Manchester, UK

K. McElreavey, Paris, France

H. Morrison, Woods Hole, MA, USA

S. Mueller, Munich, Germany

E. Murchison, Cambridge, UK

K. Nagaki, Okayama, Japan

M. Nieves, Buenos Aires, Argentina

S. Nokkala, Turku, Finland

E. Olmo, Ancona, Italy

C. Ozouf-Costaz, Paris, France

M-L. Pardue, Cambridge, MA, USA
S. Pearce, Brighton, UK

A. Pedrosa-Harand, Recife, Brazil

D. Penman, Stirling, UK

D. Phillips, Aberystwyth, UK

R. Phillips, Vancouver, WA, USA

M. Plohl, Zagreb, Croatia

J. Postberg, Witten, Germany

T. Raudsepp, College Station, TX, USA

W. Rens, Cambridge, UK

N. Royle, Leicester, UK

J. Rufas, Madrid, Spain

A. Ruiz-Herrera

R. Saffery, Parkville, Australia

K. Sahara, Sapporo, Japan

M. Sakaizumi, Nigata, Japan

H. Scherthan, Freiburg, Germany

M. Schmid, Wurzburg, Germany

Y. Shevelev, Russia

A. Simms, Edinburgh, UK

J. Siroky, Brno, Czech Republic

S. Svard, Uppsala, Sweden

V. Trifonov, Novosibirsk, Russia

F. Veyrunes, Montpellier, France

P. Waters, Canberra, Australia

M. William, San Diego, CA, USA

H. Winking, Leubeck, Germany

P. Vogt, Heidelberg, Germany

J-N. Volff, Lyon, France 